\title{
Un blog électronique pour développer la compétence lexicale en FLE
}

\author{
Recherche préparée par \\ Prof. Dr. Hussein Taha \\ Professeur de didactique du FLE, Faculté de Pédagogie, Université de Sohag

\section{Dr. Doaa Ismaël El-Kady} \\ Maître de conférences en didactique du FLE, Faculté de Pédagogie, Université \\ d'Assiout
}

\section{Azhar Mohamed Ahmed Mohamed}

Enseignante de FLE à Al-Azhar Al-Charif 


\section{Résumé}

La présente recherche vise à vérifier l'effet de l'utilisation d'un blog électronique sur le développement de quelques compétences lexicales en FLE chez les élèves de la $1^{\text {ère }}$ année du cycle secondaire à Sohag. Pour vérifier cet objectif, une méthode (quasi- expérimentale) a été utilisée avec un design d'un seul groupe expérimental $(\mathrm{N}=30)$ et une pré-post application du test de compétences lexicales. L'analyse statistique des résultats concernant le test des compétences lexicales a constaté qu'il existe une différence statistiquement significative entre la moyenne des notes de l'échantillon de la recherche au pré- application du test des compétences lexicales et celle de leurs notes à la post- application du même test en faveur de la post-application. Cela affirme l'effet positif de l'utilisation du blog proposé sur le développement de la compétence lexicale en FLE chez les membres le l'échantillon.

Mots clés : compétence lexicale, relations sémantiques fondamentales, blog.

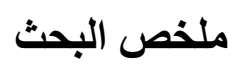

يهدف هذا البحث إلى دراسة أثر إستخدام مدونة إلكترونية على تتمية مهار ات تعلم مفردات اللغة

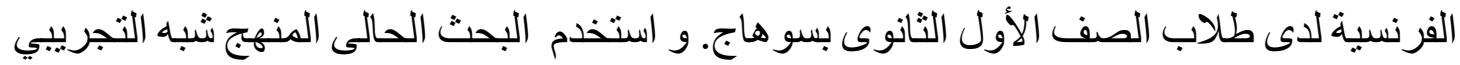

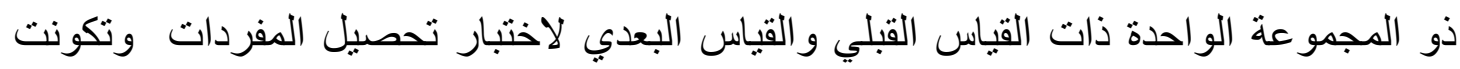

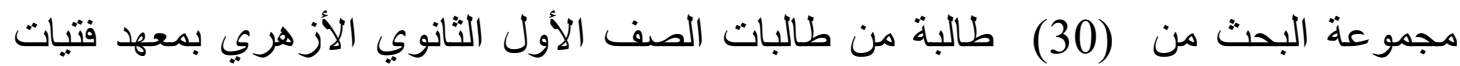

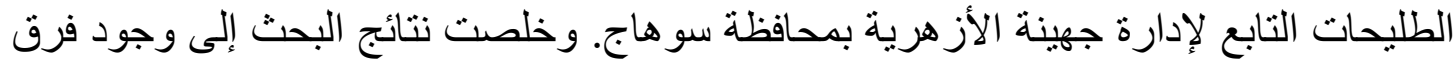

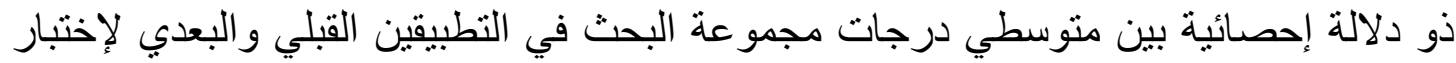

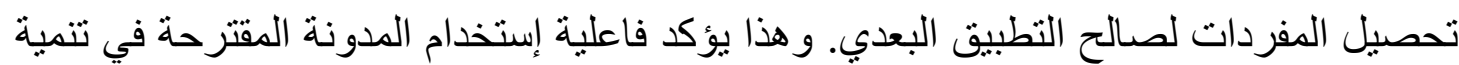
مهار ات تعلم المفردات في اللغة الفرنسية. 


\section{1- Introduction}

$\mathrm{Au}$ cours des dernières années, l'usage des nouvelles technologies de l'information et la communication (NTIC) dans l'enseignement s'est accru de façon considérable notamment dans le domaine de l'enseignement des langues étrangères. Ces nouvelles technologies, comme le constate Taha (2011, 23), constituent, aujourd'hui, des outils précieux dans les réformes de l'enseignement qui visent à développer les fameuses compétences langagières en didactique du FLE (Compréhension orale et écrite et production orale et écrite).

Or, les applications pédagogiques de (NTIC) se propagent aujourd'hui, dans le monde éducatif. Ces différents outils rendent possible un accès facile à l'information, ainsi qu'une communication plus dynamique entre enseignants ou étudiants (Barbier et Verrier, 2010,16). Plus concrètement, l'utilisation de l'Internet est considérée comme une des utilisations de ces technologies dans le domaine de l'éducation, en général, et dans le domaine de l'enseignement des langues étrangères en particulier (Barbel, 2007,33).

Avec le développement rapide de ce réseau mondial et de ses usages dans tous les domaines de la vie quotidienne, il n'est pas surprenant que les enseignants de langues sont attirés parce qu'on peut voir a priori comme un inépuisable réservoir de ressources à caractère informatif et d'outil de communication. (Mangenot et Dejean, 2009, 336).

Parmi les médias basés sur l'Internet et utilisés dans l'enseignement des langues étrangères sont le site web, l'e-mail et plus récemment le blog (Jehelen, 2011,30). Ce nouvel outil est une des premières applications du Web 2.0 qui jouit d'une popularité à grande échelle, le blogue éducatif peut constituer l'une des réussites les plus visible du web 2.0 (Camilleri, 2007, 37).

Le blogue se caractérise par une grande facilité et adaptabilité aux différents contextes didactiques de communication dans l'enseignement d'une langue étrangère. L'une des clefs du succès des blogues provient de leur simplicité (Bérubé et Poellhuber, 2006,33). N'importe qui peut créer 
son blog en quelques minutes, et l'un de ses intérêts réside dans l'interactivité qu'il propose avec les visites grâce aux commentaires donnés à chaque billet. Selon Baruch $(2006,57)$, un blog est un réel espace de communication.

Plusieurs études (Asselin, 2003, Baruch, 2006, Camilleri, 2006, Domejoz, (2006), Lanlanne 2006, Tomé, 2009, Carralet, M et Lanneau, 2007, Chirol, 2007, Lombard, 2007, Mader, 2007, Pérez, 2007, Quint, 2007, Tomé, 2007), Audet, 2010), Ngnoulayé 2010, Hocine, 2011, Koeing, 2011 et Teysseder, 2012) ont montré que le blog est l'une des applications technologiques efficaces pour développer les compétences de l'orthographe en FLE, l'autonomie en apprentissage, la production orale, la motivation scolaire. De même, ces études ont mis en évidence un lien étroit entre l'usage de ces outils et la création d'une communication authentique dans la classe de langue et, aussi, la potentialité de développer la maitrise des compétences lexicales.

De tout ce qui précède, la recherche actuelle s'appuie sur ces résultats pour vérifier l'effet d'un blogue proposé sur le développement de quelques compétences lexicales en FLE chez les élèves de la $1^{\text {ère }}$ année du cycle secondaire.

\section{2- Problématique de la recherche}

Malgré l'importance majeure des compétences lexicales, l'apprentissage de ces compétences explicite plusieurs problèmes en didactique du FLE. Certaines études ont constaté la faiblesse des compétences lexicales chez les élèves comme l'étude de (Hunkler, 2005, Rodrigues, 2005, Noureddine, 2006, Anctil, 2007, Rigaudeau 2007, Muller 2009, ChaaLuste, 2009, Lahlou, 2009, Muller, 2009, Haboul, 2010, Salinas, 2010, Bentolila, 2011, Matet, 2011 et Abdel Karim, 2012). S'ajoute à cela que l'apprentissage du vocabulaire passe souvent par un processus de traduction et que ceci est inopérable et inefficace dans l'usage adéquat des mots dans des contextes de communication variés. Salinas et Avram (2010). Ce déficit risque de perturber gravement l'apprentissage des autres compétences langagières (Bentolila, 2011). 
La chercheuse a observé pendant son travail comme enseignante de français langue étrangère à l'institut de Tolihate pour les filles à Gouhina (Sohag), que le niveau des élèves de la première année secondaire est faible à propos de la maitrise des compétences lexicales suivantes :

-Catégorisation des mots.

-Utilisation des synonymes des mots.

-Utilisation des antonymes des mots.

-Utilisation des mots homonymes.

En vue de confirmer cette observation, une étude pilote a été effectuée pour évaluer la compétence lexicale chez les élèves de la première année secondaire à l'institut de Tolihate pour les filles à Gouhina. Un test de compétences lexicales a été appliqué sur un échantillon de 25 élèves. Le tableau ci-dessous montre les résultats du test.

Tableau N (1): les résultats de l'étude pilote au test des compétences lexicales chez les members de l'échantillon

\begin{tabular}{l|c|c}
\hline Les compétences & $\begin{array}{c}\text { Le nombre des élèves } \\
\text { qui sont capables de } \\
\text { répondre aux } \\
\text { questions concernant } \\
\text { les compétences visées }\end{array}$ & $\begin{array}{c}\text { Le pourcentage de } \\
\text { maitrise }\end{array}$ \\
\hline $\begin{array}{l}\text { Connaître la } \\
\text { catégorisation des } \\
\text { mots }\end{array}$ & 8 & $32 \%$ \\
\hline $\begin{array}{l}\text { Utiliser les synonymes } \\
\text { des mots }\end{array}$ & 7 & $28 \%$ \\
\hline $\begin{array}{l}\text { Utiliser les antonymes } \\
\text { des mots }\end{array}$ & 4 & $16 \%$ \\
\hline $\begin{array}{l}\text { Utiliser les mots } \\
\text { homonymes }\end{array}$ & 4 & $16 \%$ \\
\hline
\end{tabular}


Ainsi, les résultats indiqués dans le tableau ci-dessus mettent en relief qu'il y a une certaine faiblesse dans l'acquisition de la compétence lexicale en FLE. D'après ces constats et face à cette marginalisation pour la compétence lexicale en FLE, on a essayé dans la présente recherche de tirer profit des avantages des blogs en tant que média capable de favoriser l'apprentissage et le développement de cette compétence.

\section{3- Question de la recherche}

Cette recherche tente de répondre à la question principale suivante :

"Quel est l'effet de l'utilisation d'un blog électronique sur le développement de compétence lexicale en FLE chez les élèves de la $1^{\text {ère }}$ année secondaire à Al-Azhar Al-Charif ?"

\section{4- Objectif de la recherche}

La présente recherche vise à vérifier l'effet de l'utilisation d'un bolg électronique sur le développement de la compétence lexicale en FLE chez les élèves de la première année secondaire à Al-Azhar Al-Charif.

\section{5- Hypothèse de la recherche}

L'hypothèse de la présente recherche peut être statistiquement formulée de la manière suivante:

- Il existe une différence statistiquement significative entre la moyenne des notes de l'échantillon de la recherche à la pré application du test de compétences lexicales et celle de leurs notes à la post application du même test en faveur de la post-application.

\section{6- Limites de la recherche}

La recherche actuelle se limite à :

1)-Un échantillon de 30 élèves de la première année secondaire à l'institut de Tolihate pour les filles à Gouhina.

2)-Quelques compétences lexicales, ce sont:

- Catégoriser les mots.

- Utiliser les synonymes des mots. 
- Utiliser les antonymes des mots.

- Utiliser les mots homonymes.

3)-Appliquer l'expérimentation proposée en deuxième semestre de l'année scolaire 2017/2018.

\section{7- Méthodologie de la recherche}

La recherche actuelle vise, comme nous l'avons déjà souligné, à identifier l'effet de l'utilisation du blog électronique sur le développement de la compétence lexicale en FLE chez les élèves de la $1^{\text {ère }}$ année du cycle secondaire à Sohag. Pour vérifier cet objectif, une méthode (quasiexpérimentale) a été utilisée avec un design d'un seul groupe expérimental et une pré-post application du test.

\section{8- Outils de la recherche}

Les instruments suivants ont été élaborés:

1. Une liste de quelques compétences lexicales.

2. Un blog électronique.

3. Un guide de l'enseignant

4. Un pré /post test de compétences lexicales en FLE.

\section{9- Procédures de la recherche:}

Pour répondre à la question de la recherche, on a suivi les étapes suivantes :

\section{(1) Pour le cadre théorique :}

-Consulter les études et les recherches antérieures qui concernent l'usage des blogs et la maitrise de la compétence lexicale en FLE.

\section{(2) Pour le cadre expérimental:}

-Elaborer une liste de quelques compétences lexicales et la présenter au jury afin de savoir leurs points de vue en ce qui concerne les compétences lexicales que les élèves de la $1^{\text {ère }}$ année du cycle secondaire doivent 
maîtriser et les compétences nécessaire aux élèves de la $1^{\text {ère }}$ année secondaire.

-Elaborer le contenu du blog en suivant quelques étapes :

A. Déterminer les objectifs généraux et spécifiques du blog.

B. Préciser les différentes sources du blog.

C. Préparer le contenu du blog.

D. Elaborer les activités pratiquées dans le blog.

E. Présenter le contenu du blog au jury (spécialistes en sciences du langage et en méthodologie du français langue étrangère) et le modifier dans sa forme finale.

-Elaborer le pré-post test des compétences lexicales pour détecter jusqu'à quel point les élèves de la $1^{\text {ère }}$ année secondaire maîtrisent-ils les compétences lexicales.

-Choisir un échantillon composé de 30 élèves de la $1^{\text {ère }}$ année du cycle secondaire.

-Appliquer le pré-test sur l'échantillon de la recherche (le groupe expérimental).

-Appliquer le blog proposé sur l'échantillon de la recherche.

-Appliquer le post-test sur l'échantillon de la recherche.

-Vérifier l'hypothèse, analyser statistiquement les résultats obtenus et les interpréter.

- Présenter les recommandations et les suggestions générées de la recherche.

\section{0- Cadre Théorique}

\section{Axe (1)- Le vocabulaire:}

10-1 Importance de la maitrise de la compétence lexicale dans l'enseignement/apprentissage du FLE

Jusqu'aux années 70 le vocabulaire est un aspect secondaire soumis à la grammaire et il est appris au moyen des listes des mots (Monge, 2013:439). 
Depuis les années 80 , le vocabulaire a recommencé à occuper une place importante en didactique des langues étrangères et l'on admet désormais que les mots sont le pivot de la langue (Bogaards, 1994: 143; Tréville \& Duquette, 1996: 10). Ils sont indispensables pour communiquer et leur manque constitue un grand obstacle tout aussi bien pour comprendre que pour s'exprimer. Depuis quelques années, les chercheurs montrent que pour s'exprimer correctement en langue étrangère, une bonne connaissance du vocabulaire est indispensable dans la communication. C'est-à -dire, les connaissances lexico-sémantiques sont, du point de vue de la communication, plus importantes que la syntaxe surtout aux premiers stades de l'apprentissage et elles sont essentielles pour tout acte langagier. Ainsi, Bogaards $(1994,39)$ affirme qu' « il est tout à fait possible d'utiliser à bon escient un mot sans se servir de sa syntaxe». Comme le montre Nguyen $(2004,2)$ «une fois que l'apprenant possède une bonne connaissance lexicale, il pourrait toujours finir par se débrouiller dans beaucoup de cas difficiles».

Par ailleurs, le vocabulaire peut être considéré comme une des composantes fondamentales et essentielles d'une langue et de son acquisition. Ce sont les mots qui formulent les langues, ou en d'autres termes, sans mot, pas de langue. Comme l'ont montré Tréville et Duquette $(1996,9)$ qu' "apprendre une langue c'est essentiellement apprendre le vocabulaire de cette langue". On voit alors qu'une bonne acquisition du vocabulaire permet d'explorer la langue dans toute sa profondeur dans ses aspects linguistiques aussi bien que pragmatique. Comme l'a mentionné David $(2000,31)$ la place privilégiée du vocabulaire en tant que l'une des composantes les plus importantes de l'acquisition des langues sur tous les versants: en compréhension, comme en production, à l'oral comme à l'écrit.

Ainsi, nous nous trouvons désormais dans une période qui admet que «le lexique, haut porteur d'information, contribue, avec l'intonation, à donner rapidement aux élèves l'accès à la communication » (Courtillon 1989, cité par Bogaards, 1994, 9). Ce qui exige qu'en classe de langue se réalise un enrichissement lexical continu et soigné chez l'apprenant. 
De plus, l'étude de Desvaux (2008) a montré que l'enseignement du vocabulaire est une nécessité à tout apprentissage. Egalement, Isabelle $(2008,9)$ a confirmé que la maîtrise du vocabulaire est le point de départ pour maîtriser une langue. D'ailleurs, comme l'a déjà montré Claude (2006, 20), s'il est très important de maîtriser la langue sur le plan grammatical, il est encore plus primordial d'avoir une bonne connaissance du vocabulaire. Ce dernier constitue l'une des composantes les plus importantes de l'acquisition du langage, sur tous versants : en compréhension comme en production, à l'oral comme à l'écrit. Plane (2007, 33) constate la corrélation entre réussite scolaire et connaissances lexicales. Sabourin $(2009,95)$ va dans le même sens en ajoutant que le vocabulaire est le facteur principal qui différenciait l'apprenant fort de faible. Dralet $(2007,9)$ a fait observer qu'il est impossible de développer une pensée rigoureuse ni de communiquer avec autrui, sans aucun vocabulaire. Thuan $(2009,15)$ partage le même résultat en constant que le vocabulaire est depuis longtemps considéré comme "la chair de la langue". En effet, il est indispensable d'enseigner le fonctionnement d'une langue qui serait vide de sens, vide de mot, le mot est le pivot autour duquel s'organisent les syntaxes grammaticales. Comme l'a dit Marie $(2000,23)$ qu'on peut communiquer sans avoir aucune connaissance en grammaire, mais certainement pas sans posséder du vocabulaire.

Pour conclure, on peut dire que le vocabulaire est l'axe principal dans l'enseignement / apprentissage d'une langue étrangère. Il est, comme le dit Cuq et Grusa $(2002,361)$ pour l'apprenant comme le canal le plus direct qui relie à son système conceptuel. C'est pour cela, l'entrée par vocabulaire est une tendance presque naturelle à tout apprenant d'une langue.

\section{0-2 Les relations sémantiques fondamentales}

Les relations sémantiques qui vont être présentées ici sont considérées comme étant les relations sémantiques "fondamentales" car elles forment, comme le souligne Polguère $(2002,120)$ la charpente de la structuration sémantique du lexique de toute langue. Chaque lexie se positionne dans le réseau lexical de la langue tout d'abord en fonction de ces relations. De 
plus, on peut constater qu'à l'intérieur du lexique, il y a des mots qui entretiennent entre- eux des rapports sémantiques plus ou moins étroits. Certains entretiennent entre-eux des relations d'identité (les synonymes) ou d'opposition (les antonymes), ou d'inclusion (les hyponymes), d'autres encore se ressemblent au niveau formel (les homonymes).

\section{0-2-1 Les synonymes}

Ce sont des vocables qui forment une relation sémantique fondée sur une similarité de signifiés entre des signifiants différents. Elles désignent également la relation entre deux ou plusieurs formes différents (deux ou plusieurs signifiants) ayant le même sens (un seul signifié) entretiennent entre-elles. En principe, on établit la synonymie en utilisant une procédure de substitution: on remplace un mot par un autre dans le même contexte (Salminen, 1997, 110)

De plus, l'interchangeabilité des synonymes n'est pas dans n'importe quel contexte: "battre" et "frapper" peuvent être synonymes dans le groupe "battre / frapper quelqu'un" mais ils ne le sont pas dans "battre un tapis" où "frapper" ne peut pas se substituer à "battre". Pour cela, il est nécessaire d'adapter le synonyme au contexte. Comme l'a montré Bogaards (1994,32), la synonymie est un phénomène contextuel, c'est l'emploi en contexte qui doit décider de la synonymie ou de la non-synonymie de deux unités lexicales.

C'est pourquoi, avant de remplacer un mot par un synonyme, il faut donc analyser le contexte parce qu'un même mot peut avoir, selon le contexte donné et le sens dans lequel il est utilisé, des synonymes différents. Par exemple, le mot «vieux » signifie «âgé » dans la phrase « Mon grand-père a 95 ans, il est très vieux (âgé) », mais le même mot signifie «ancien» dans la phrase «Nous habitons dans une vieille (ancienne) maison avec une grande cave ». Nous ne pouvons pas échanger le mot «ancien » et le mot « âgé » dans ces deux phrases même s'ils sont les synonymes du même mot « vieux». 
Il est important de souligner que la synonymie parfaite n'existe pas, deux UL n'ont jamais exactement le même sens (Dumarest, 2009,24). On le voit surtout dans les expressions figées où la synonymie est bloquée, il est impossible de remplacer souliers par chaussures dans (être dans ses petits souliers) cela veut dire (être embarrassé), cela pour des raisons sémantiques et souvent historiques.

\section{0-2-2 Les antonymes}

Ce sont des mots qui s'opposent directement l'un à l'autre par le sens. C'est-à-dire, ils se distinguent par leurs signifiants, et de sens contraire.

L'antonymie apparait d'une certaine façon comme le contraire de la synonymie. Comme le souligne Salminen (1997, 113), elle désigne une relation entre deux termes de sens contraires. Il est important de souligner que les mots mis en opposition doivent avoir en commun quelques traits qui permettent de les mettre en relation de façon pertinente. La relation d'antonymie existe surtout dans les mots qui représentent des qualités ou des valeurs (bon / mauvais, long / court, grand / petit, ...etc., de déplacements (haut / bas, devant / derrière, droite / gauche), des rapports chronologiques (jeune / vieux).

\section{0-2-3 Les hyponymes}

L'hyponyme est un lexème subordonné à autre lexème, qui lui est superordonné, et qui est appelé hyperonyme (Neveu, 2009:54). Par exemple, animal est un hyperonyme de chien et que chien est un de ses hyponymes, avec d'autres lexies telles que chat, cheval, dromadaire, poisson, ... etc. (Polguère, 2002, 120).

D'ailleurs, l'hyponymie ne caractérise pas seulement la catégorie nominale, elle se manifeste, comme le souligne Kleiber $(1990,128)$ dans d'autres classes de mots, notamment les qualificatifs, les verbes. Par exemple, rédiger / libeller / écrire. L'hyponymie est donc une relation sémantique d'inclusion entre deux lexèmes $\mathrm{Ce}$ rapport de sens entre 
signifiés lexicaux, défini en terme d'inclusion sémantique, renvoie au sens d'une unité lexicale, implique dans celui d'une autre. Par exemple, le sens de rose est inclut dans celui de fleur, mais l'inverse n'est pas vrai.

La loi de réciprocité, ici, ne s'applique pas. La rose est une partie des fleurs, ainsi que le matérialise de cette structure : toute rose est une fleur. Une telle structure d'appartenance traduit, comme l'a déjà cité Nisubire (2001, 37-38), une implication unilatérale, qui va dans le sens de l'hyponyme à l'hyperonyme, une relation hiérarchique qui régit fleur et ses autres hyponymes, tels que tulipe, jasmin, lilas,...etc.

De plus, la relation d'hyperonymie- hyponymie d'après Polguère (2002: 121) est transitive et permet donc de construire une hiérarchie sémantique des lexies, hiérarchie qui peut se représenter sous la forme d'un arbre :
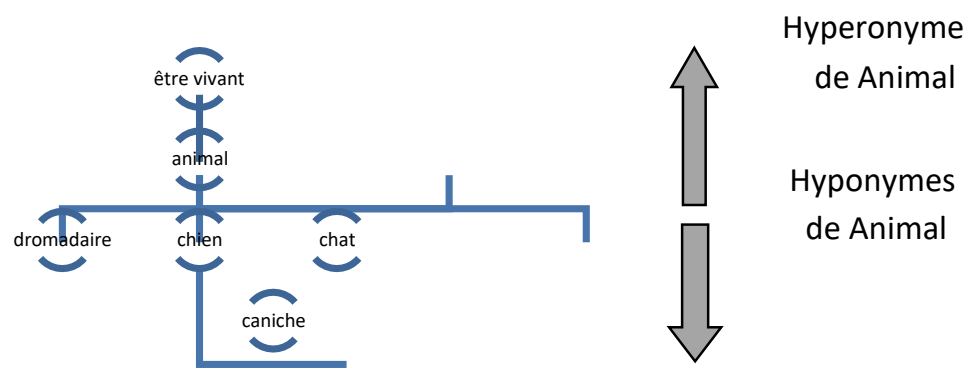

D'après la figure ci-dessus, si Animal est un hyperonyme de chien et chien un hyperonyme de caniche, alors Animal est aussi un hyperonyme de caniche.

En effet, les relations hyperonymiques et hyponymiques ont donc un rôle central dans l'enseignement/apprentissage du lexique dès l'instant où elles nous permettent d'élaborer des classes d'unités lexicales qui répartissent leurs liens sémantiques du sens le plus général au plus particulier (Dumarest et al.,2009,27).

\section{0- 2-4 Les homonymes}

Tout d'abord, deux mots se trouvent dans la relation d'homonymie lorsqu'ils présentent un signifiant identique et des signifiés distincts. Elle désigne la relation de similarité de signifiants entre des formes 
linguistiques dont les signifiés sont différents, cette similarité peut être tout à la fois d'ordre phonique et graphique (homophonie et homographie), c'est-à-dire, une homographie peut coïncide avec une homophonie. Par exemple: car (conjonction) et car (moyen de transport) sont deux homophones homographes (Neveu, 2009,51).

Par ailleurs, la relation de similarité peut être d'ordre exclusivement phonique par exemple : maire et mère sont deux homophones hétérographes. Elle peut être d'ordre exclusivement graphique. Par exemple : fils (Fis), dans fils unique, et fils (fil), dans il a arraché les fils, sont deux homographes hétérophones. C'est-à-dire, qu'ils soient homographes ou simplement homophones, les homonymes ont, comme l'a montré Touratier $(2000,87)$ dans les deux cas, un signifiant identique, et des signifiés différents.

\section{$\underline{\text { Axe (2)-Les blogs }}$}

\section{0-2 Vue historique du blog:}

Il est facile de deviner que l'éclatement des blogues est fortement lié à l'avènement d'Internet. Ainsi, selon Desavoye (2005), le phénomène du blog accompagne la croissance fulgurante de l'utilisation d'Internet, sa vulgarisation et son intégration à nos habitudes quotidiennes.

A l'origine, les blogs d'après Garreau $(2005,4)$ étaient des listes de liens commentés, surtout produites et utilisées par des scientifiques. Le premier blog aurait été, ainsi, la page d'accueil de Tim Berners Lee en 1989, sur laquelle le créateur du Web indiquait chaque jour de nouveaux liens et de brefs commentaries. En 1997, John Barger, ingénieur américain et chercheur en intelligence artificielle invente le terme weblog. L'outil de publication des blogues "blogger" en 1999 voit le jour. Ce site permet à quiconque de créer facilement un blogue en remplissant un court formulaire d'inscription. (Bachand, 2009)

En effet, les blogues sont nés à la fin des années 1990 et ils ont connu une percée bien plus importante après les attentats du 11 septembre en 
2001. Plusieurs internautes américains y ont trouvé un moyen d'évacuer le traumatisme émotionnel engendré par cette catastrophe. Et pour discuter les événements se déroulant autour de la guerre lancé par les Etats-Unis contre le terriorisme en Irak en 2003. On peut ajouter des événements qui viennent toucher la blogosphère, comme le Tsunami en Asie, l'élection de Barack Obama, les élections en Iran et d'autres événements qui ont été significatifs dans la blogosphère. Bref, c'est à l'occasion d'évènements internationaux majeurs que les blogosphères se développent. (Barbeau, 2011,15).

Le blog est devenu en quelque temps un véritable phénomène. Comme le montre Fafard (2009), il représente, aujourd'hui, le plus répandu mode de partage des pensées personnelles. Le blogue est facile à éditer et à publier en ligne, sans recours au langage de programmation.

\section{0-3 Les avantages du blog}

\section{0-3-1 Simplicité}

Tous les médias sociaux sont relativement faciles d'utilisation, mais le blogue est probablement le média le plus simple de mettre en ligne des contenus. Comme le dit Vandal (2006), "Montre en main, il ne faut pas plus de cinq minutes pour créer un blogue".

En effet, la diffusion et l'une des clefs du succès des blogues provient de leur simplicité de création et de publication. N'importe qui peut créer son blogue en quelques minutes. Heureusement, millions de personnes qui créent et animent quotidiennement des blogs ne sont pas tous experts en informatique (Falque - Pierrotin, 2006).

D'ailleurs, les modifications faites sur la page par le bloggeur sont effectuées automatiquement par le système qui présente au bloggeur sa page modifiée. Aucune programmation n'est pas nécessaire pour l'utilisation d'un blogue. Comme le souligne Lombard (2005, 8), la facilité de mise en œuvre et d'emploi d'un blog donne à chacun la possibilité de présenter ses idées au monde entier et suscite les réactions des internautes. 


\section{0-3-2 L'interactivité}

Le blogue est un espace interactif, contrairement à une page web classique .Il propose un système de rétroaction offrant aux visiteurs la possibilité de commenter les divers billets publiés par l'auteur du blogue.

En effet, la force du blogue réside en particulier dans l'interaction possible entre l'auteur et les lecteurs / commentateurs. Autrement dit, tout nouvel entrant dans une communauté de la blogosphère doit établir une relation de confiance au sein d'un groupe (Pledel, 2007, 9).

Le blogue permet une interactivité entre le créateur du blogue et ses lecteurs. Ceux-ci ont, en effet, la possibilité de réagir aux messages de l'auteur, d'apporter leur avis sur le blogue en général, ou de lire les réactions des autres visiteurs. Les interactions peuvent alors prendre l'aspect d'une discussion entre l'auteur et le lecteur (Lalancette, 2010, 8).

\section{0-3-3 Multimédia}

En effet, le multimédia est une œuvre qui combine plusieurs éléments tels que textes, sons, images fixées, images animées, programmes informatiques dans un même support. On connait deux types de multimédia: hors ligne et en ligne. L'internet est un exemple de multimédia en ligne.

D'ailleurs, le multimédia est l'utilisation de divers éléments pour établir un milieu interactif. Depuis longtemps, il est resté au service de l'enseignement/apprentissage des langues étrangères. C'est un outil pour apprendre en s'amusant. Une des particularités du multimédia d'après Fafard $(2009,44)$ est de faciliter le transfert d'information.

\section{0-3-4 Interconnexion}

Les blogues contiennent de multiples liens vers d'autres sources d'information sur la toile des sites web, et aussi, d'autres blogues. Il se crée ainsi une sorte de maillage entre blogues. 
D'ailleurs, le RSS également contribue au succès des blogues. Le Rss, les blogues, wikis ou toute autre plateforme du web 2.0 sont mis à jour constamment, de façon très simple et n'importe où. Pour faciliter aux visiteurs la visualisation de toutes ces mises à jour. Le web 2.0 est fondé sur les flux Rss. De son côté falque-pierrotin (2006) souligne que RSS est une abréviation de Really Simple Syndication, "Syndication Vraiment Simple", ou de Riche Site Summary,"Sommaire d'un site enrichi", ou il s'agit d'un système technique qui permet au blogueur de récupérer automatiquement des informations publiées sur un autre blog.

\section{0-3-5 La personnalisation}

Personnaliser l'apprentissage, c'est tenir compte du style et du rythme d'apprentissage de la personne, de ses préférences, des conditions, des modes d'apprentissage, de ses aptitudes, de la motivation à apprendre, etc. (Sauvé, 2014).

En effet, l'éducation et la formation tout au long de la vie grâce aux technologies web et aux applications pédago-technologies permettent aux dispositifs en ligne de personnaliser l'apprentissage aux besoins de chacun.

En effet, le web même plus collaboratif, évolue une plus grande personnalisation. C'est à la fois des contenus qui sont d'avantages représentatifs d'un individu (profil, photos, opinion, etc.), et de leur organisation par cet individu qui choisit et combine les ressources en ligne pour y avoir accès selon ses intérêts et ses préférences.

La particularité du blogue repose contrairement aux médias traditionnels, comme le signale Klein (2008,7), sur le blogueur ou l'auteur du blogue qui possède seulement la possibilité d'effectuer les actions de réduction, de révision, de mise en ligne et l'administration 


\section{1- Instruments de la recherche et ses procédures}

\section{1-1- Elaboration du test de compétences lexicales}

- Objectif du test: le test utilisé lors de cette recherche a visé à mesurer l'effet de l'utilisation d'un blog électronique sur le développement de quelques compétences lexicales en FLE chez les élèves de la première année secondaire à $\mathrm{Al}$ Azhar $\mathrm{Al}$ Charif.

-Après avoir élaboré le test sous sa forme initiale, on l'a présenté au members du jury en leur demandant de déterminer si:

Le test est au niveau des élèves de la première année secondaire.

Le test est pertinent et compatible pour mesurer les compétences lexicales visées.

Modifier les questions mal formées.

-Appliquer le test: On a appliqué le test avant et après l'expérimentation pour vérifier son efficacité.

\section{1-2- Elaboration du blog des compétences lexicales}

Le blog proposé a été élaboré en suivant les étapes suivantes :

- Créer un compte sur gmail (compétenceslexicales@gmail.com).

- Créer le blog sur le site www.blogger.com sous l'adresse suivante:www.compétenceslexicales.blogspot.com

- Créer une chaîne sur youtube (compétenceslexicales).

- Créer un compte sur www.powtown.com (multimedia140).

- Préparer les activités en utilisant le programme "Quiz creator".

- Désigner les pages des modules.

- Télécharger les activités sur le compte google drive puis la chercheuse les a Téléchargé sur le blog.

- Préparer les pages du blog.

- Relier les pages des blog.

- Déterminer le type de la navigation dans le blog en cliquant sur n'importe quelle icône pour commencer la navigation. 


\section{1-3-Le contenu proposé du blog}

Le contenu proposé a comporté quatre modules avec huit leçons:

Module (1) a comporté trois leçons.

Module (2) a comporté deux leçons.

Module (2) a comporté une seule leçon.

Module (2) a comporté deux leçons.

Chaque leçon a comporté:

Les compétences visées à développer, le contenu de la leçon sous une forme d'une vidéo (powtown), les activités de la leçon à télécharger sur n'importe quel lieu dans l'ordinateur et l'évaluation de la leçon(les élèves ont laissé la réponse à la question de l'évaluation dans les commentaires).

\section{1-4-Elaboration d'un guide d'utilisation du blog}

On a préparé un guide en vue d'aider les élèves à utiliser facilement chacune des composantes du blog.

\section{1-5-Expérimentation}

Après avoir élaboré le contenu du blog, on a commencé l'étude expérimentale en suivant les démarches suivantes:

\section{1-5-1- Choix de l'échantillon de la recherche:}

On a choisi un seul groupe expérimental composé de 30 élèves de la première année secondaire à l'institut "Tolihate" pour les filles à " Gouhina" à Sohag.

\section{1-5-2-Application du pré-test sur les members de l'échantillon de la recherche:}

Après avoir déterminé l'échantillon de la recherche et avant de commencer l'application de l'expérimentation, on a appliqué le test des compétences lexicales comme pré- test sur l'échantillon de la recherche le 27 juillet 2018.

\section{1-5-3-Déroulement de l'expérimentation:}

Le schéma de la démarche suivie dans le déroulement de l'expérimentation didactique le second semestre lors de l'année scolaire 2017/2018 se présente comme suit:

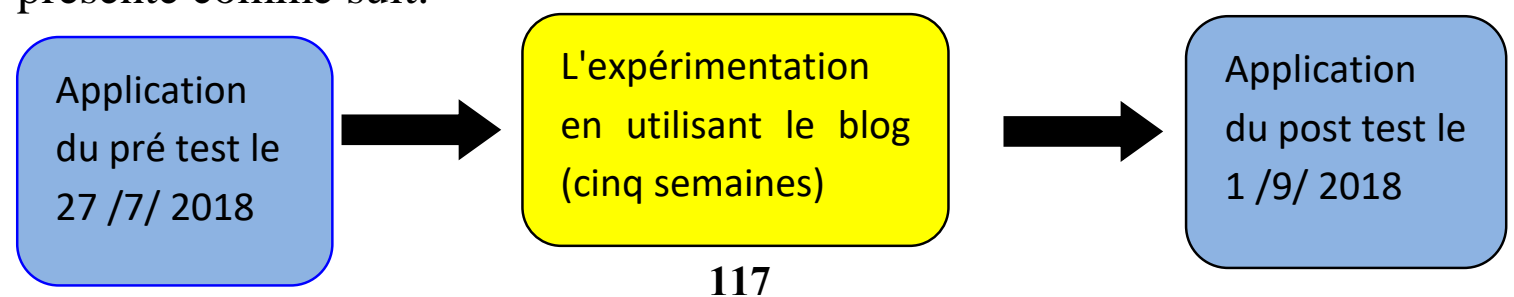




\section{1-5-4-La passation du blog proposé:}

L'expérimentation a débuté le 28 juillet 2018 et s'est terminé le 30 août 2018. Elle a donc durée presque cinq semaines. L'un des auteurs du texte a dirigé le contenu du blog aux élèves de la première année secondaire à l'institut de Tolihate pour les filles à Gouhina.

\section{1-5-5-L'application du post-test:}

Après avoir fini l'expérimentation, on a successivement appliqué le post -test aux membres de l'échantillon de la recherche le premier septembre 2018.

En ayant des résultats avant et après l'expérimentation, pour mesurer l'effet d'un blog électronique sur le développement de quelques compétences lexicales en FLE chez les élèves de la première année secondaire à $\mathrm{Al}$ Azhar $\mathrm{Al}$ Charif. On va présenter dans les lignes suivantes l'analyse statistique de ces résultats concernant le test des compétences lexicales.

\section{1-5-6-Traitements statistiques des données}

Pour vérifier l'hypothèse de la présente recherche selon laquelle "il existe une différence statistiquement significative entre la moyenne des notes de l'échantillon de la recherche au pré application du test des compétences lexicales et celle de leurs notes à la post application du même test en faveur de la post-application', nous avons calculé les valeurs suivantes:

Tableau N (2): la valeur de (T) au pré-post test des compétences lexicales chez les membres des l'échantillon

\begin{tabular}{l|l|l|l|l|l|l|l}
\hline $\mathrm{N}$ & $\begin{array}{l}\text { Moyenne } \\
\text { des notes } \\
\text { au pré } \\
\text { test }\end{array}$ & $\begin{array}{l}\text { Moyenne } \\
\text { des notes } \\
\text { au post } \\
\text { test }\end{array}$ & $\begin{array}{l}\text { Dégré } \\
\text { de } \\
\text { liberté }\end{array}$ & $\begin{array}{l}\text { Valeur } \\
\text { de T }\end{array}$ & $\begin{array}{l}\text { Valeur } \\
\text { de } \eta^{2}\end{array}$ & $\begin{array}{l}\text { Carré } \\
\text { d'écart } \\
\text { type des } \\
\text { moyennes }\end{array}$ & $\begin{array}{l}\text { Seuil de } \\
\text { significativité }\end{array}$ \\
\hline 30 & 15.6 & 43.8 & 29 & 54.05 & 0.990 & 236.8 & Significative \\
\hline
\end{tabular}


En regardant le tableau ci-dessus, on remarque que la moyenne des notes des membres de l'échantillon au pré -test est de 15.6 et la moyenne des notes au post - test est de 43.8 et la valeur de $\mathbf{T}$ est de 54.05 et la valeur de $\eta^{2}$ est de 0.990. Cela indique qu'il y a une différence statistique significative entre la moyenne des notes de l'échantillon au pré/post test des compétences lexicales en faveur de post- test. On déduit que le blog proposé a une grande influence sur le développement de quelques compétences lexicales chez les élèves de la première année secondaire.

Egalement, pour bien étayer nos résultats, nous avons calculé les valeurs de $\mathbf{T}$ et $\eta^{2}$ pour chacune de quatre compétences visées par la recherche actuelle. Le tableau suivant montre bien ces valeurs:

Tableau N (3): les valeurs de (T) pour les quatre compétences visées au pré-post test des compétences lexicales chez les membres de l'échantillon

\begin{tabular}{l|c|c|c|c|c|c|c}
\hline Compétence & $\begin{array}{c}\text { Moyen } \\
\text { ne des } \\
\text { notes } \\
\text { au pré } \\
\text { test }\end{array}$ & $\begin{array}{c}\text { Moyenne } \\
\text { des notes } \\
\text { au post } \\
\text { test }\end{array}$ & $\begin{array}{c}\text { Dégré } \\
\text { de } \\
\text { liberté }\end{array}$ & $\begin{array}{c}\text { Valeu } \\
\text { r de T }\end{array}$ & $\begin{array}{c}\text { Valeu } \\
\text { r de } \\
\eta 2\end{array}$ & $\begin{array}{c}\text { Carré } \\
\text { d'écart } \\
\text { type des } \\
\text { moyennes }\end{array}$ & $\begin{array}{c}\text { Seuil } \\
\text { de } \\
\text { signific } \\
\text { ativité }\end{array}$ \\
\hline $\begin{array}{l}\text { Catégoriser } \\
\text { les mots }\end{array}$ & 6.8 & 17 & 29 & 30.26 & 0.969 & 98.8 & \\
\hline $\begin{array}{l}\text { Utiliser les } \\
\text { synonymes } \\
\text { des mots }\end{array}$ & 1.9 & 8.1 & 29 & 38.29 & 0.980 & 22.8 & \\
\hline $\begin{array}{l}\text { Utiliser les } \\
\text { antonymes } \\
\text { des mots }\end{array}$ & 2.6 & 9.1 & 29 & 34.15 & 0.975 & 31.5 & signific \\
\hline $\begin{array}{l}\text { Utiliser les } \\
\text { mots } \\
\text { homonymes }\end{array}$ & 4.2 & 9.9 & 29 & 35.60 & 0.977 & 22.3 & ative \\
\hline
\end{tabular}

D'après le tableau précédent, on remarque que les valeurs de $\mathrm{T}$ sont de 30.26 pour la catégorisation des mots, 38.29 pour l'utilisation des mots synonymes, 34.15 pour l'utilisation des antonymes des mots et 35.60 pour l'utilisation des mots homonymes. Toutes ces valeurs sont significatives au niveau de 0.01. Pour bien étayer cette différence significative, nous avons 
calculé la taille de l'effet, on peut observer que les valeurs de $\eta^{2}$ sont: (0.969- 0.980- 0.975- 0.977). L'hypothèse de la recherche a été bien confirmée. On déduit donc que le blog proposé a une grande influence sur le développement de la compétence lexicale en FLE chez les élèves de la première année secondaire.

\section{1-5-7 Discussions et interprétations}

A la lecture des tableaux ci-dessus, il est apparaît clairement que l'hypothèse suggérée par la présente recherche a été acceptée. En observant les valeurs indiquées dans les tableaux (2), ces valeurs mettent en évidence qu'il existe une différence significative au pré-post test des compétences lexicales en faveur de post -test. Comme le montre ce tableau, la moyenne des notes des membres de l'échantillon de la recherche était au pré-test 15.6 et est devenue au post-test 43.8 ; il nous parait donc que les notes de l'échantillon étaient faibles au pré-test. Cela signifie que les élèves éprouvaient de grands problèmes concernant de déterminer les catégories des mots, identifier le mot intrus, classifier les mots en catégories, connaître les synonymes des mots, remplacer les mots par leurs synonymes, connaître les antonymes des mots, identifier le sens des mots homonymes dans un contexte et formuler des phrases simples avec des mots homonymes. Elles ne s'entrainaient pas suffisamment aux activités à propos de celles-ci. L'utilisation des sources électroniques en ligne dans le blog comme les dictionnaires en ligne: dictionnaire pour les synonymes, pour les antonymes et pour la traduction (la rousse) pour traduire d'une langue à l'autre les a aidé à développer leur motivation en vue de réaliser les objectifs de l'apprentissage.

Aussi qu'en observant le tableau (3), on remarque que les valeurs de T sont statistiquement significatives et la moyenne des notes des members de l'échantillon dans chacune de quatre compétences visées au pré/test et au post/test sont significatives. Cela indique qu'il y a une différence significative entre les moyennes des notes de l'échantillon au pré/test et celle du post/test dans l'amélioration de quatre compétences lexicales. 
Pour conclure, on peut dire que l'utilisation du blog électronique pour développer la compétence lexicale chez les élèves de la première année secondaire, était efficace grâce aux points positifs suivants:

- La variété des sources d'apprentissage (textes, images, vidéos) et les activités variées présentées tout au long du déroulement de l'expérimentation ont contribué au développement de la motivation des élèves pour l'apprentissage et de la qualité de leurs compétences en acquisition du vocabulaire.

- Le blog où se trouve le contenu proposé qui se compose de quatre modules, était disponible tout le temps aux élèves. En outre, le fait d'être en ligne les encourageait à profiter de toutes les ressources électroniques y intégrées.

- La disponibilité de la formatrice, soit face à face dans la laboratoire d'ordinateurs de l'institut pendant l'application du blog, soit en ligne à travers le commentaire dans le blog avait une grande influence sur l'interaction entre elle et les élèves. Ce qu'avaient confirmé Costa et Hernandes (2011), les blogs représentent des outils dynamiques qui favorisent la création et la motivation chez les apprenants, ainsi qu'une Communication authentique, ils peuvent renforcer le travail collaboratif en classe.

- Les sites utiles intégrées à mon blog ont offert aux élèves la chance de pratiquer plusieurs types d'activités et la recherche des informations sur ces sites leur a donné la chance de l'autoapprentissage, ce qu'avait confirmé Chirol (2007).

- Le feedback immédiat que les élèves ont obtenu après avoir fini la réponse à chaque question leur ont offert la chance d'évaluer leurs réponses en leur donnant immédiatement la réponse correcte.

- Le blog a donné aux élèves la possibilité de télécharger les activités pourqu'elles peuvent exercer hors ligne si elles ne sont pas connectées à l'internet dans n'importe quel temps.

- Le feedback de la formatrice sur les commentaires des élèves leur a permis d'évaluer elles-mêmes. 


\section{Bibliographie}

- Abdel-karim, M. (2012). "Efficacité d'un programme proposé basé sur l'approche éclectique pour développer la compétence lexicale chez les étudiants du cycle secondaire". Thèse de magistère. Institut des études pédagogiques. Université du Caire.

- Allard, D. (2008).Connaissances lexicales en morphologie dérivationnelle chez les apprenants avancés en français langue seconde. Thèse de magistère. Université du Québec à Montréal.

- Alain, P. (2002). Notions de base en lexicologie. Université de Montréal. Canada. Disponible sur: http: //www.fas.umontreal.ca/ling/olst.

- Anctil, D. (2012).Portrait des erreurs lexicales d'élèves de $3^{\text {ème }}$ année secondaire en production écrite et proposition de pistes didactiques: Pratiques ,155-156, 251-256.

-Asselin, M. (2003). Apprentissage des langues et Réseaux médias sociaux. Disponible sur : http://flenet.rediris.es/blog/carnetweb.html. -Audet, L. (2010). Wikis, blogues et web2.0 opportunités et impacts pour la formation à distance, le réseau d'enseignement francophone à distance du canada(REFAD).Disponible sur: http://www.refad.cu/nouveau/wikisblogues-et-web2.0.pdf.

-Barbier. etVerrier, C. (2002).Internet, l'imaginaire et l'enseignement universitaire en ligne. Conférence au CIES-Sorbonne, 23 Janvier, université Paris 8-laboratoire de l'éducation et cultures (CRISE).

- Bentolila, A. (2007). Rapport de mission sur l'acquisition du Vocabulaire à l'école élémentaire. Disponible sur : http://www.Education-duVocabulaire-a-l-ecole-elementaire.html.

- Bérubé, B.et, Poellhuber, B. (2005).Un référentiel de compétences technologiques. Disponible sur:

http://eureka.ntic.org/file_download.php?lom_id=2761\&filename=refernt iel.pdf.

- Bogaards, P. (1994). Le vocabulaire dans l'apprentissage des langues étrangères.Paris: Hatier

- Cadre européen commun de référence pour les langues : apprendre, enseigner, évaluer (CECR) (2001).Disponible sur: http://www.coe.int/t/dg4/linguistic/cadre_fr.asp. 
- Calaque, E. (2009). Construction du vocabulaire et Construction des Connaissances au Cours moyen. LIDILEM. Université de Stendhal. Grenoble III. Disponible sur http://www.Calaque.airdf.fse.ulaval.Ca/fichier/communicationcalaque.pdf.

- Camilleri, M. (2007).Blog dans l'enseignement des langues vivantes. Centre européen pour les langues vivantes. Disponible sur: http://archives.ecml.at/mtp2/D-Blog-F-Internet.pdf.

- Carralet, M et Lanneau, P. (2007).Un blog comme outil transversal de formation et de suivi pour les étudiants en mobilité à l'étranger. Les actes du colloque du RIFEFE.8-10 novembre.

-Chaa, O, L. (2009).L'acquisition lexicale en français langue étrangère: conceptions et applications. Thèse de magistère. Université Stendhal.Grenoble 3.France.

-Chirol, S. (2007).Un blog: pourquoi? Pourquoi? Bilan et perspectives. Colloque cyber-langues-perpignan 12 Septembre. Disponible sur: http://www.cyber-langues.asso.fr/spip.php. article19?

-Claude, A- J. (2006). La mémorisation du Lexique. Disponible sur: http://www.crdp-Montpellier.fr/resources/memoire/200616-86booob.pdf. -Crapel, H.H. (1999). Compétence lexicale et acquisition / apprentissage. Cahier de l'ASDFLE, 6.Actes des 13 e et 14e Rencontres. Paris, Janvier Septembre.

- Cuq., J.P\& Gruca., I. (2002). Cours de didactiques du français langue étrangère et seconde. Grenoble: Presses universitaires.

- Cuq., J.P. (2003). Le dictionnaire de didactique du français langue étrangère et seconde. Paris: CLE international.

-Darmawangsa, D. (2007).Le blog dans l'enseignement de la production écrite. Cédille Revesta d'estudios franceses, 3, 265-268.Disponible sur: http://file.upi.edu/LE-BLOG-DANS-L'ENSEIGNEMENT.pdf.

- Degach, C. et Garbarino, S. (2012). Enseignement / apprentissage du lexique: apprendre-entre-les-langues dans un contexte roumanophone. Acte du colloque IC2012.Université Stendhal. Grenoble 3.France. 
- Desvaux, P. (2008). La Compétence lexicale au service de la lecture. Construire le vocabulaire un apprentissage sur le long terme. Disponible sur:http://www.lettres.ac-aix-marseille.fr/college/longue-

lexique/2008.pdf.

-Domenjoz, J-C et Lachat, C. (2006).Blog à l'école .Rapports d'activités. P297-325.Service Ecole-Médias Disponible sur: http://icp/sem/pdf-blog2rapport-06-07.pdf.

- Dralet, S. (2007). Apprentissage de la lecture et vocabulaire. Comment un enrichissement lexical permet - il d'améliorer les Compétences des apprentis - lecteur? Disponible sur : http://www.ac-reims.Sylviedralet.pdf.

- Gachet, S. (2008). Savez - vous jouer aux charades ? Approcher la grammaire et le vocabulaire par les charades. Disponible sur : http://www.prof-fle.com/prof-fle/charades.pdf.

- Giasson, J. (2008). La Compréhension en lecture. De Boeck université. Disponible sur: http://signeset.eu/fr/acte-lexique/jGiasson-Compr-1.

- Haboul, D. (2010).Compétence dérivationnelle et stratégies de communication. Cas d'élèves du secondaire. Synergies Algérie.11, 25-83. - Hocine, N. (2011).Intérêts pédagogiques de l'intégration des TICE dans l'enseignement du F.L.E.L'utilisation du web-blog dans des activités de production écrite. Synergies Algérien 12, 219-226.Disponible sur: http://ressources-cla-univ-fcomte.fr/naima/hocine.pdf.

- Hunkeler, H. (2005). L'évolution de la didactique du vocabulaire dans L'enseignement primaire et secondaire. Disponible sur: http://www.amazon.jeux-eneignementvocabulaire/dp/2866225953/Publibook.com/boutique2005.datailu-2134pb.html.

- Ivan, S. (2008).Retenir mieux des mots nouveaux par l'apprentissage incident ou intentionnel au cours de la lecture. Disponible sur:http://www.er.uqam.ca/12170/retenir_mieux.html.

- Jehlen, S. (2011). L'enseignement /apprentissage des langues étrangers à l'heure du web 2.0 et des réseaux sociaux. Thèse professionnelle. In MBA spécialisés et formation continue du pôle universitaire léonard de Vinci. 
- Kleiber, G. (1990). La sémantique du prototype. Catégories et sens lexical. France: Presses universitaires.

- Koeing, A. (2007).Le blogs et didactique des langues étrangères. Disponible sur:http://fle, asso.free.fr/liens/blog.html.

- Lahlou, S. (2009). La place de l'enseignement du vocabulaire dans les classes de FLE du secondaire hellénique : attitudes et pratiques des enseignants. Synergies sud - est européen. 2. $37-43$.

- Lalanne, G. (2010). L'utilisation des TICE dans le cadre d'un projet de correspondance. Institut universitaire de formation. Disponible sur : http://www.cudp.fr/savoirs-cdi/auteurs/lalanne.pdf.

- Lombard, F. (2007).Blogs et Wikis: actualité de la didactique ou didactique de l'actualité? Disponible sur:http://tecfa.unide.ch/perso/lombardf/publications/actualiteouvrage/wiki_blogs_actualie_de_didactique.pdf.

-Mader, C. (2007). Technique d'écriture coopérative par le blog. Disponible sur : http://www.lanouvellepublic.frcharlotte-la-specialiste.

-Mangenot, F. et Dejean- Thircuir, C. (2009).Modalités de communication pédagogique dans la formation en ligne. In Cannelas-Trévise et al. (dir), Langage, objets enseignés et travail enseignant, 335-351.Grenoble, Ellug. - Mario, J. (2011). L'apprentissage lexical en français langue étrangère: théories d'acquisition et pratique de classe. Disponible sur : http://www.lecafedufle.fr/apprentissage-lexical-en-FLE.

- Matet, D. (2011).Les compétences lexicales et lecturales de jeunes adolescents en grande difficulté scolaire cas des élèves SEGPA. Thèse de doctorat .Université Paris Descartes.

- Mortureux., M.F. (1997). La lexicologie entre langue et discours. Paris: SEDES.

- Muller, F. (2007). L'enseignement du vocabulaire dans les manuels. Université de paris x-Nanterre. Disponible sur : http://www.ebims.org/archive/amullero.pdf.

- Ngnoulayé, J. (2010).Étudiants universitaires du Cameroun et les technologies de l'information et de la communication: usage, apprentissage et motivation. Thèse de doctorat. Faculté des sciences et de l'éducation. Université de Montréal. 
-Nisubire, P. (2001). La compétence lexicale en français langue seconde. Stratégies et activités didactiques. Editions Modulaires Européennes. Collections proximités-Didactiques. Intercommunications.S.P.R.L.

- Noureddine, B.(2011).L'enseignement/apprentissage du vocabulaire du FLE dans le secondaire: problèmes et percepectives. Thèse de magistère, faculté des lettres et des langues. Université Mentouri-Constantine.

-Pérez, C. (2007). Le web 2.0 dans l'enseignement des langues étrangères. Disponible

sur:http://carmenvera.wikispace.com/Le+Web+2,0+Dans+L'enseignemen t+Des langues+\%C3\%A9trang.pdf.

-Pierrotin, F, I. (2006). Je blogue tranquille. Disponible sur: http://www.foruminternet.org.ddn/guide-blog-net-pdf.

- Plane, S. (2004). Un Consensus à interroger ; la question des «mots difficiles » Quelques éléments d'une enquête sur le repérage des problèmes lexicaux. Actes du 9e Colloque de L'AIRDF. Québec .De 26 au 28 Août. IUEM. Paris V.

- Rigaudeau, L. (2007). Le lexique en classe de $6^{\mathrm{e}}$ (présentation, mémorisation, appropriation).Disponible sur: http://grammle.ulb.ac.be/fichiers/colloques/Nantes2007/PESCHEUX.pdf.

-Robert, P. (2010). L'occurrence des mots dans la rétention du lexique : Cas du manuel de 3ème année primaire. Disponible sur : http://www.univ.chlef-dz/paul-Robert/CommunicationMissoum.2010.pdf.

-Rodrigues, C. (2005). Aide à l'apprentissage du vocabulaire dans un environnement hypermédia en français langue étrangère. Thèse de doctorat. Université Blaise pascal. Clermont II.

- Salminen, N. (1997). La lexicologie. Paris: Armand Colin.

- Salinas, A. et Avram, C. (2010). Représentations sémantico-lexicales et hypothèses de traduction en Roumain pour des mots inconnus en français langue étrangère et langue des affaires. Synergies Roumanie, no5,pp. 107119.

- Sylvie, S. (2003). Enseignement du lexique : approche empirique. Disponible sur : http://www.crdp-Montpellier/resources/memoiresmemories/2003/b/0/03b0048/03b5548.pdf. 
- Taha, H. (2011).Impact de l'Internet sur le développement de la compréhension orale chez les étudiants de la faculté de pédagogie de Sohag. Association égyptienne de Curricula et de Méthodologie. 171, 23 65.Juin.

- Tchernigosvkaïa, M. (2008). La compréhension et le réemploi du vocabulaire suite à un enseignement par ordinateur à l'aide des définitions versus et des Concordances. Thèse de Maîtrise. Université du Québec. Montréal.

- Teyssedre, G. (2012).Quels obstacles à l'integration des TICE dans l'enseignement élémentaire. Thèse de doctorat. Université Bordeau2.Disponible sur : http://www.theses.fr/2012/BOR21915.pdf.

- Thierry, L. (2006).Internet, usages et innovation: quelle intégration en cours de FLE? Les cahiers de l'Asdifle. 17,23-35.Disponible sur: http://www.ciep.fr/internet/usage/et/innovation/THIERRY.pdf.

- Thuan, N. Q. (2009). Enseigner le vocabulaire selon l'approche Communicative. Séminaire régional de recherche - action Nha - trang.

Disponible : http://refef.crfef.crifpe.Ca/document/nhatrang/33\%25\%N25 $\%$ Q250\%.thuan.

- Tomé, M. (2009).Weblogs éducatifs pour l'enseignement d'une langue. Synergies Espagne, no2, pp.261-279.

- Trémion, V. (2005).Le blog, un outil pour l'apprentissage du FLE. Journée de l'Asdifle, 22 octobre. Disponible sur: http://flenet.rediris.es/tourdetoile/Vtremion-blog.pps.

- Tréville., M.C. \& Duquette, L. (1996). Enseigner le vocabulaire en classe de langue. Paris: Hachette.

- Triquenot, A. (2007). Incidence de la préfixation sur le comportement sémantique. Thèse de doctorat. Université Paris Descartes.

- Vigner, G. (2007)."Le monde, les mots et l'école. Élément d'une didactique du vocabulaire à l'école élémentaire". Repères no 193.PP191209. Disponible sur : http://ife.ens-lyon.fr/publication/INRP-RS00812.pdf.-

- Wisniewska, K-A. (2011).Stratégies de collaboration pour un interactif en FLE dans une communauté virtuelle d'élèves blogueurs. Thèse de doctorat. Université d'Aix-Marseille. 